Received: December 21, 2016 Accepted: January 10, 2017 Published: January 30, 2017

\title{
A Rare Case of Newborn with Accessory Scrotum Asso- ciated with Bifid Scrotum and Perineal Lipoma
}

\author{
Enrica Rossi ${ }^{1,2, *}$,Massimo Basile $^{1}$,Ubaldo Bongini $^{2}$,Anna Maria Buccoliero ${ }^{3}$,Chiara \\ Caporalini ${ }^{3}$,Claudio Defilippi ${ }^{1}$ \\ ${ }^{1}$ Department of Radiology, Meyer Children's Hospital, Florence, Italy. \\ ${ }^{2}$ Department of Imaging, Bambino Gesù Children's Hospital. Piazza Sant'Onofrio 4, \\ Rome Italy. \\ ${ }^{3}$ Pathology Unit, Meyer Children's Hospital, Florence, Italy.
}

*Corresponding author: Enrica Rossi MD, Department of Imaging, Bambino Gesù Children's Hospital. Piazza Sant’Onofrio, 4 Rome, Italy,Tel: 06/68592790; E-mail: enrica.rossi@opbg.net

[1-4].

Neonates presenting with perineal masses should undergo further imaging and evaluation to rule out additional congenital deformities.

\section{Abstract}

We report a case of bifid scrotum with accessory scrotum and peduncular lipoma in perineal region occurring in a full-term male neonate. Physical examination showed two soft perineal masses located between a bifid scrotum and the anus. No abnormalities of anus were detected. The patient underwent ultrasound and magnetic resonance examinations confirming a homogeneous fat tissue matter of the posterior mass and showing fluid content inside the anterior one. The patient also underwent a Gastrografin enema and no analcolon anomalies were detected.

The masses were completely excised and the histological examination revealed a lipoma, with tissue suggestive of scrotum, so a definite diagnosis of accessory scrotum, associated with lipoma was made.

\subsection{Keywords}

accessoryscrotum;bifidscrotum;perineallipoma; newborn hamartoma

\section{Introduction}

Neonatal perineal masses are very unusual. We report a rare case of accessory scrotum (AS) with peduncular lipoma. AS is the least frequent perineal abnormality, with only 43 cases reported in the English literature [1]. AS is characterized by additional scrotal tissue lacking a testis, besides a normally developed scrotum. Various associated anomalies have been reported. In particular, contiguous subcutaneous tumor is the most frequently associated abnormality and it is reported to be related to the etiology of AS

\section{Case report}

A full-term male neonate (3 days old) with external perineal anomalies was referred to our Hospital. He was born after a regular pregnancy at gestational age of 39 weeks, via spontaneous vaginal delivery having cephalic presentation. His birth weight was 3280 gr and his Apgar index was 9-10. The mother was healthy with no family history of congenital abnormalities.

The physical perineal examination revealed a bifid scrotum containing palpable testis and a normal configured penis located at the bottom of the bifid scrotum. Two soft masses of 3 and $2 \mathrm{~cm}$ respectively, divided from a cutaneous notch, were located below the bifid scrotum and on the right of the midline. The rear biggest mass was normal epithelized, instead the other one was a rugged pigmented mass, which resembled the scrotum (Figure 1). There were no additional abnormalities of the external genitalia.

A patent anus was seen, slightly located on right side. The child presented with fecal and urine content, passing meconio autonomously. US examination showed a hyperechoic solid tissue, corresponding to the rear biggest perineal mass. The other peduncolar mass, located between the right scrotum and the posterior mass, had fluid content (Figure 1A, B). A mild hydrocele in the right scrotum and a sliding testis on the left side were also revealed.

A Gastrografin enema was performed to evaluate the rectum and colon and tested negative (Figure $1 \mathrm{C}, \mathrm{D}$ ).

MRI also confirmed two perineal peduncolar masses: the biggest and posterior one, was made up by homogeneous fatty matter without contrast-enhancement after intravenous gadolinium injection (Figure 1E, F, G), whereas the other one, showing muscles signal intensity wall and a little amount of fluid content, demonstrated contrast enhancement post-Gad (Figure $1 \mathrm{H}, \mathrm{I}$ ).

The patient underwent excision of perineal masses and no com- 

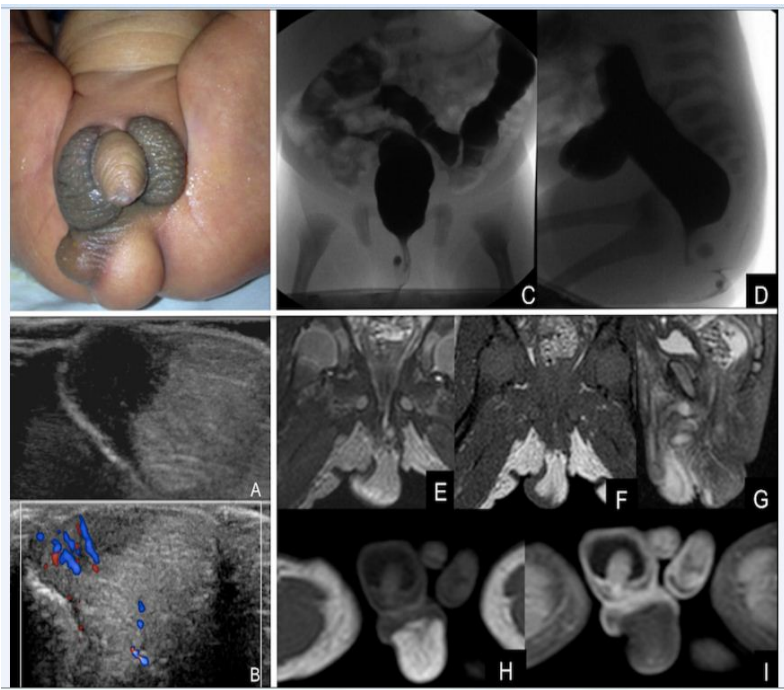

Figure 1: The physical perineal examination revealed a bifid scrotum and a normal configured penis, with two soft masses below measuring 3 and $2 \mathrm{~cm}$ respectively. The rugated and pigmented skin of the anterior protuberance was contiguous with the covering normal skin of the main posterior mass. There were no additional abnormalities of the external genitalia and anal sphincter was normal.

Ultrasonography examination of the rear biggest protuberance showed an oval-shaped soft mass with similar echogenicity to the adjacent subcutaneous fat, instead the anterior protuberance appeared as a round-shaped mass with fluid content. B. Doppler US demonstrated vascularization only inside the wall of the anterior rugated mass. Single contrast-study of lower gastrointestinal tract shows the colon in its normal position, regularly filled by the contrast $(C)$, and it proves also normal diameter of rectum $(D)$.

Coronal T2-weighted $(E)$, T1-weighted $(F)$ and sagittal fat suppressed T2-weighted $(G)$ magnetic resonance imaging view of the pelvis showing the homogeneous fatty matter of the biggest rear mass, and the muscles signal intensity wall of the anterior mass. The sagittal fat suppressed T2-weighted $(G)$ also showed little amount of fluid content inside the anterior mass. Axial fat suppressed T1-weighted view $(H, I)$ demonstrated contrast enhancement after gadolinium injection of the anterior mass $(I)$.

plications occurred in the surgery.

The histopathological examination of the perineal masses revealed two areas with different histological features: the first one was characterized by the presence of smooth muscle bundles dispersed in the dermal collagen, instead the other contiguous area showed abundant mature adipose tissue in the deep dermis and hypodermis (Figure 2). At last the rugged swelling mass was definitively diagnosed as AS without testis tissue inside, and the rear mass was diagnosed as lipoma.

\section{Discussion}

Neonates presenting with perineal masses are uncommon. There are very few cases recorded in literature with genital abnormalities presenting perineal masses [1].

Congenital abnormalities of the scrotum are uncommon and include accessory, or ectopic or bifid scrotum and penoscrotal transposition. Among these, AS is the least frequent with only 43 cases reported in the most recent review [1]. AS is characterized

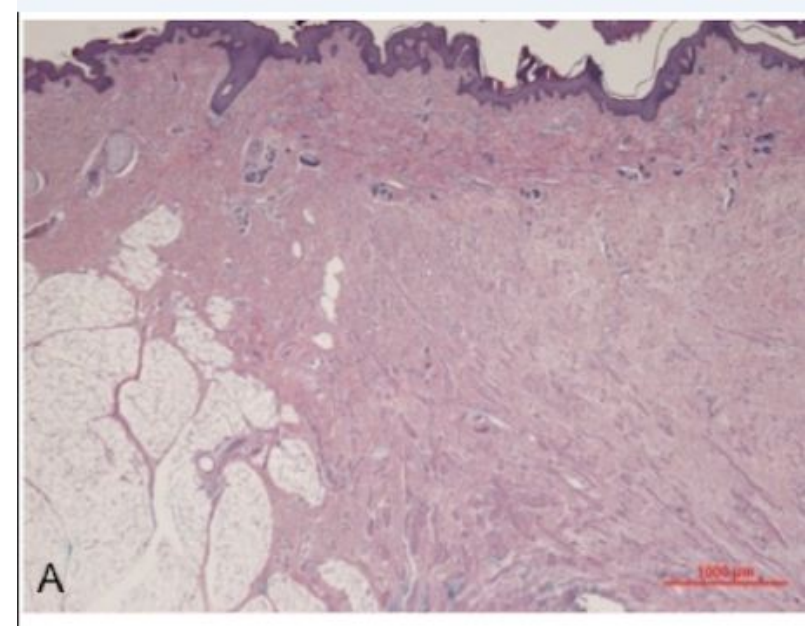

(a)

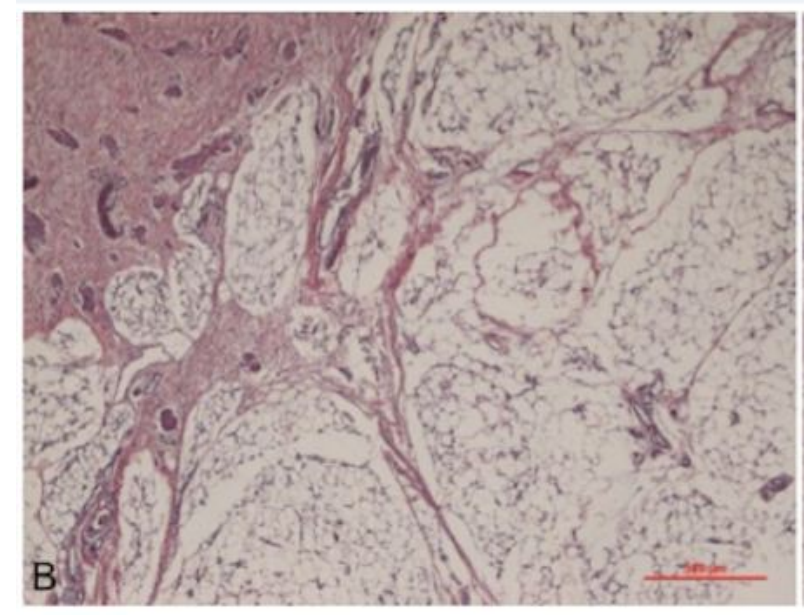

(b)

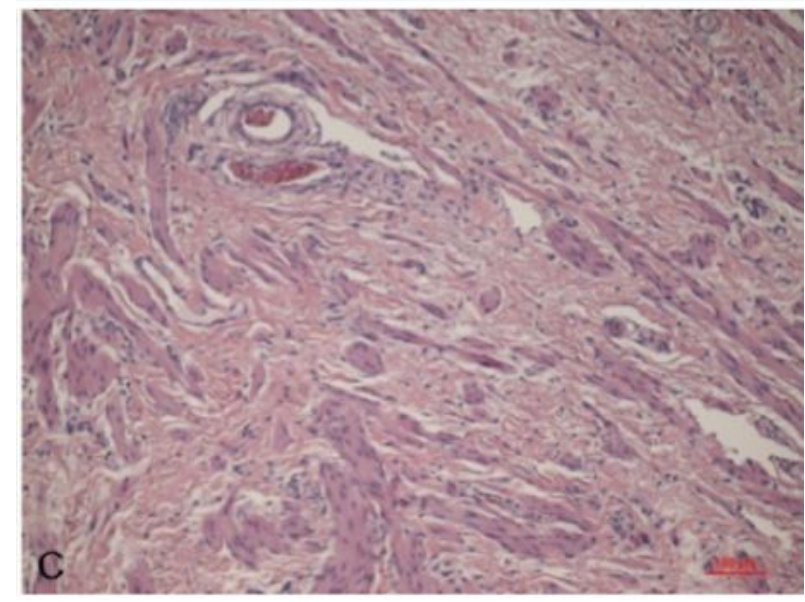

(c)

1 Figure 2: Surgical specimen consisted of cutaneous and subcutaneous tissue showing two areas with different histological features: an area characterized by the presence of smooth muscle bundles dispersed in the dermal collagen and a contiguous area with abundant mature adipose tissue in the deep dermis and hypodermis (H\&E magnification Ax20; Bx40; Cx100). 
by additional scrotal tissue lacking a testis. A normal scrotum is usually developed with normal testis inside.

These anomalies can occur isolated or more rarely in combination with other abnormalities such as uro-genital or ano-rectal anomalies [1] or with contiguous subcutaneous tumors [1,2]. A recent review showed that these 43 cases of tumors consisted in: 39 cases of lipoma, 1 case of lipoblastoma, 3 cases of hamartoma [1]. It is also reported that these tumors could be related to the etiology of AS [1].

Various hypotheses have been developed to explain the association of these pathologies. It has been postulated that in human embryos the normal development of the urogenital system and anorectum unit may depend on the integrity of the mesenchymally- derived urorectal septum [3].

Scrotal development starts at 4 weeks' gestation with the appearance of paired labioscrotal swellings lateral to the cloacal membrane that migrates inferomedially and merges at 12 weeks to form the scrotum. Abnormal migration of the inferior portion of these swellings may result in an accessory scrotum. Sule and colleagues hypothesized that AS develops when intervening mesenchymal tissue disrupts the continuity of the developing caudal labioscrotal swelling [3], instead Takayasu hypothesized that AS develops from the early division and teratoid growth of pluripotential labioscrotal tissue elements in order to explain the association with no contiguous tumor [4].

During the period of scrotal development, migration of the genital folds medially forms the perineal raphe from the midpoint of the anus to the edge of the glans. Thus, arrest in migration results in anocutaneous, ano-urethral or anobulbar fistulae $[3,4]$.
Furthermore when patients develop hipotrofic perineal musculature, both urinary and bowel continence may be affected.

Finally, literature proves that detection of perineal masses is possible with careful prenatal screening [4,5]. The differential diagnosis of a fetal perineal mass includes lipoma, lipoblastoma, infantile hemangioma, hamartoma, and choristoma [5].

In conclusion when perineal masses are found, with prenatal diagnosis or during a newborn physical examination, it is important to look for any associated congenital anomalies or subcutaneous tumors by using imaging.

\section{References}

1.Murase N, Uchida H, Hiramatsu K. Accessory scrotum with perineal lipoma diagnosed prenatally: case report and review of the literature. Nagoya J Med Sci. 2015;77(3):501-506. 2. Villarreal DH, Ortiz VN, Iturregui JR, Suarez G, Duran N. Bifid scrotum, perineal hamartoma and high imperforate anus: a case report. Bol Asoc Med P R. 1998;90(4-6):93-94.

3. Sule JD, Skoog SJ, Tank ES. Perineal lipoma and the accessory labioscrotal fold: an etiological relationship. J Urol, 1994;151(2):475-477.

4. Takayasu H, Ueno A, Tsukada O. Accessory scrotum: a case report. J Urol, 1974; 112: 826-827.

5. Ahn KH, Boo YJ, Seol HJ, Park HT, Hong SC, Oh MJ,et al. Prenatally detected congenital perineal mass using 3D ultrasound which was diagnosed as lipoblastoma combined with anorectal malformation: case report. J Korean Med Sci. 2010;25(7):1093-1096. Doi: 10.3346/jkms.2010.25.7.1093. 